\title{
Entropic interactions between fluctuating twin boundaries
}

\author{
Dengke Chen, Yashashree Kulkarni* \\ Department of Mechanical Engineering, University of Houston, Houston, TX 77204, USA
}

\begin{abstract}
Nanotwinned metals have opened up exciting avenues for the design of high-strength, highductility materials owing to the extraordinary properties of twin boundaries. The recent advances in the fabrication of nanostructured materials with twin lamella on the order of a mere few atomic layers call for a closer examination of the stability of these structural motifs, especially at high temperatures. This paper presents a study of the entropic interaction between fluctuating twin boundaries by way of atomistic simulations and statistical mechanics based analysis. The simulations reveal that fluctuations of twin boundaries are considerably enhanced in the presence of adjoining twin boundaries as their spacing, $d$, decreases. In addition, the theoretical analysis shows that fluctuating twin boundaries indeed exhibit an attractive entropic interaction which enhances their thermal fluctuations and that the entropic force decreases as $1 / d^{2}$. This finite temperature interaction between twin boundaries is fundamentally distinct from the well-known repulsive entropic interaction followed by fluctuating lipid membranes as well as many crystalline membranes and interfaces. This rather surprising attraction between fluctuating twin boundaries is attributed to their shear coupled normal motion.

Keywords: Coherent twin boundaries, Thermal fluctuations, Entropic interactions, Molecular dynamics, Shear coupled grain boundaries
\end{abstract}

\section{Introduction}

Nanostructured materials have gained prominence owing to the exciting array of physical properties that are primarily governed by the high density of interfaces and interfacial

\footnotetext{
${ }^{*}$ Corresponding author

Email address: ykulkarni@uh.edu (Yashashree Kulkarni)
} 


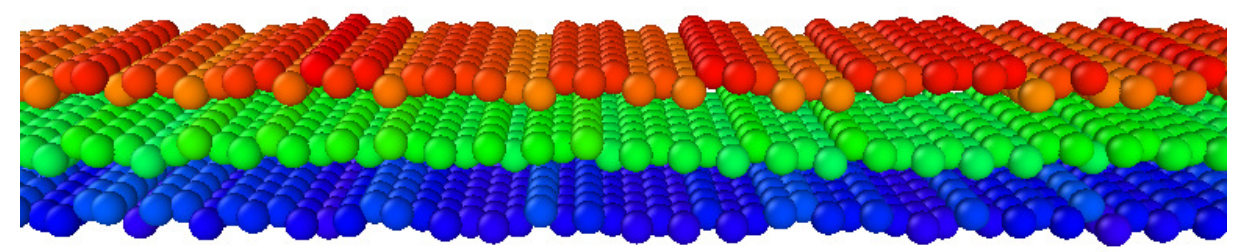

Figure 1: Atomistic structure of a section of the nanotwinned specimen depicting parallel coherent twin boundaries fluctuating at $800 \mathrm{~K}$. Only the atoms with non-zero centrosymmetry parameter (comprising the twin boundaries) are shown.

phenomena. For example, nanocrystalline face-centered-cubic metals, with an average grain size less than $100 \mathrm{~nm}$, witnessed an intensity of research in the nineties owing to their ultrahigh strength compared to their conventional polycrystalline counterparts (Gleiter, 1989; Zhu et al., 2009). However, this early enthusiasm was met with severe disappointment due to their brittle nature and loss of grain stability attributed to grain boundary (GB) mediated processes such as sliding and migration (Dao et al., 2007). In contrast, research over the past decade has provided compelling evidence that a novel class of materials known as the nanotwinned metals may be the optimal motifs for the design of high-strength high-ductility materials (Lu et al., 2004; Zhang et al., 2006; Hodge, 2008). Indeed, Lu et al. (2004) showed that nanotwinned $\mathrm{Cu}$ containing twin lamella of about $35 \mathrm{~nm}$ thickness exhibits a yield strength over $1 \mathrm{GPa}$ with elongation to failure as high as $14 \%$, which is in sharp contrast to nanocrystalline $\mathrm{Cu}$ having a yield strength of about $400 \mathrm{MPa}$ and elongation to failure of about $2-3 \%$ for comparable grain sizes. Owing to these superior properties, nanotwinned metals have been the subject of active research in recent years (Dao, 2006; Lu et al., 2009; Kulkarni and Asaro, 2009; Guo and Xia, 2011; Li et al., 2010; Jang et al., 2012; Wang et al., 2013; Mirkhani and Joshi, 2014; Wright et al., 2014).

Nanotwinned fcc metals are designed by the introduction of coherent twin boundaries (CTBs) within ultra-fine crystalline metals having a grain size of a few hundred nanometers. The typical twin lamella thickness within each grain ranges between 20-100 nm. Studies performed till date reveal that the CTBs have a very high shear strength compared to most GBs and are also effective barriers to dislocation motion leading to a Hall-Petch type strengthening mechanism (Kulkarni et al., 2009; Lu et al., 2009). However, a unique feature of the CTBs is that the twin planes are also slip planes for fcc metals which enables them 
to accommodate large plastic strains by absorption of dislocations thus enhancing ductility (Dao, 2006). In addition, experimental studies have revealed even more promising characteristics such as enhanced creep response, good thermal stability, and radiation response owing to the presence of both twin and grain boundaries (Bezares et al., 2012; Demkowicz et al., 2011; Yu et al., 2013). Very recently, Jang et al. (2012), and Wang et al. (2013) have even fabricated nanotwinned nanowires with twin spacing on the order of a few angstroms. The smallest twin lamella has only two atomic layers separating adjacent twin boundaries. Through combined experimental and computational studies, Wang et al. (2013) have shown that these nanowires with ultra-high density twins exhibit yield strength close to the theoretical strength of the metal. While these superior properties certainly open up exciting avenues for the applications of nanotwinned materials, they also call for a critical examination of the stability of these structural motifs especially at high temperatures. By structural instability we imply processes such as grain growth, which are intimately connected to properties like interfacial stiffness, mobility, as well as the interaction of the constituting interfaces, such as GBs and TBs. An analysis of the thermal fluctuations based on statistical mechanics provides a convenient and powerful approach to gain insight into these interfacial properties.

There is a rich literature on the statistical thermodynamics of membranes which was primarily developed for soft materials- fluid and polymerized membranes- that exhibit large fluctuations (Safran, 2003; Nelson et al., 2004). Based on the equilibrium thermal fluctuations, membrane theory enables estimates for quantities such as bending rigidity, and surface tension. In recent years, the membrane theory has also been applied to study crystalline membranes such as graphene and other two-dimensional materials (Los et al., 2009; Gao and Huang, 2014), as well as crystalline interfaces, such as GBs, and solid-liquid interfaces (Hoyt et al., 2001, 2006, 2010) based on thermal fluctuations. In the case of solid interfaces, the central idea is that the energetic cost for the out-of-plane fluctuation of the interface, which naturally involves deformation of the adjoining bulk regions, is used to construct an energy associated only with the interface. Then the surrounding bulk can be neglected and the interface is regarded as a membrane with an appropriate elastic energy. For instance, several high angle GBs in fcc metals exhibit capillarity induced fluctuations. This means that they can be modeled as membranes whose out-of-plane fluctuations are governed by 
surface tension, or more precisely, interfacial stiffness.

Studies based on thermal fluctuations have also shown that membranes exert a repulsive entropic pressure on each other, due to steric effects, that depends on the inter-membrane distance (Helfrich, 1978; Janke and Kleinert, 1987; Bachmann et al., 2001; Freund, 2012, 2013; Hanlumyuang et al., 2014). Unlike the extensive work on the steric interaction of membranes, the effect of the presence of multiple GBs on their thermal fluctuations has not been addressed before. This becomes more relevant in the light of the recent fabrication of nanotwinned metals with twin boundary spacing on the order of angstroms. Motivated by the striking resemblance between a stack of fluctuating biomembranes and an array of parallel fluctuating twin boundaries (Fig. 1), we seek to elucidate the entropic interaction between fluctuating twin boundaries constituting a nanotwinned structure at finite temperature.

The rest of the paper is organized as follows. Section 2 provides a review of the theory of equilibrium thermal fluctuations applied to fluid membranes and different types of crystalline membranes and interfaces. The modeling of entropic interaction between multiple membranes and the effect of this entropic force on their fluctuation spectrum is also briefly discussed. Section 3 presents the molecular dynamics simulations of the thermal fluctuations of multiple CTBs as a function of twin boundary spacing. Section 4 presents the statistical mechanics based analysis of the entropic interactions between fluctuating CTBs. The summary and potential ramifications of these findings are discussed in Section 5.

\section{Brief review of equilibrium thermal fluctuations}

We begin by providing a brief review of the statistical mechanics of membranes and how the thermal fluctuation spectra for different membranes and interfaces are related to their interfacial properties.

\subsection{Fluctuation spectrum of a free membrane}

For a fluctuating fluid membrane (Fig. 2), the energetic cost for the out-of-plane fluctuations arises from the bending rigidity of the membrane. To understand this, we first treat the membrane as a quasi-one dimensional interface that is thin in one direction, such that the fluctuation along this axis can be ignored (Fig. 3). Let $b$ be the width of the thin dimension 


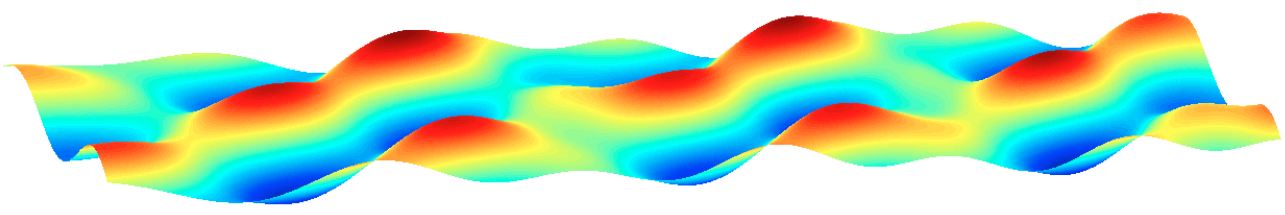

Figure 2: Schematic diagram of a freely fluctuating membrane. Different colors indicate different out of plane displacements.

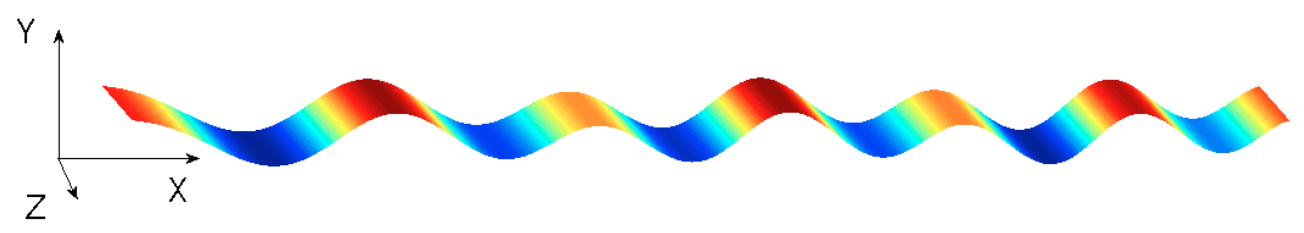

Figure 3: A fluctuating membrane approximated as quasi one-dimensional with $b \ll W$, where $W$ is the length in the direction of wave propagation (X axis), and $b$ is the width in the Z-direction. Different colors indicate different out of plane displacements.

and $W$ be the length of the longer dimension, and $h(x)$ be the out-of-plane displacement. Then the Hamiltonian can be written as

$$
\mathcal{H}=b \int_{0}^{W} \frac{1}{2} \kappa\left[h^{\prime \prime}(x)\right]^{2} d x
$$

where $\kappa$ is the bending modulus, and $h^{\prime \prime}(x)$ is the mean curvature. Expanding $h(x)$ in Fourier space,

$$
h(x)=\sum A(k) e^{i k x}
$$

the Hamiltonian can be expressed in the form

$$
\mathcal{H}=\frac{1}{2} b W \kappa \sum_{k} k^{4} A^{2}(k)
$$

This energy is quadratic in $A(k)$. Using equipartition of energy, we obtain the relationship between the mean square fluctuation spectrum and the wave vector as

$$
\left\langle A^{2}(k)\right\rangle=\frac{k_{B} T}{b W \kappa k^{4}}
$$


where $\langle\cdot\rangle$ represents ensemble average. Lipid bilayers, graphene and other two-dimensional materials are known to follow this relation. The fluctuations of many GBs and crystalmelt interfaces can also be modeled in a similar fashion. Previous studies showed that the fluctuations of high angle GBs and solid-liquid interfaces in fcc metals can be described using the capillarity or the surface tension model as (Hoyt et al., 2001, 2010)

$$
\left\langle A^{2}(k)\right\rangle=\frac{k_{B} T}{b W \Gamma k^{2}}
$$

where $\Gamma$ is the grain boundary stiffness analogous to the surface tension. However, not all GBs exhibit capillarity induced fluctuations. A recent study revealed that the fluctuations of GBs that undergo shear-coupled normal motion, such as many low-angle GBs, display a significantly different response (Karma et al., 2012). Shear-coupled motion refers to the motion of a GB normal to the GB plane when subjected to shear deformation parallel to the GB plane. Using this property exhibited by low-angle GBs, Karma et al. (2012) derived a modified expression for their thermal fluctuations as

$$
\left\langle A^{2}(k)\right\rangle=\frac{k_{B} T}{b W C \beta^{2} k} .
$$

Here, $C$ is a parameter comprising of the elastic constants of the materials, and $\beta$ is a coupling factor defined as

$$
\beta=v_{\|} / v_{n}
$$

Introduced by Cahn et al. (2006), $\beta$ characterizes the coupling relationship between the velocity parallel to the GB $\left(v_{\|}\right)$and the concomitant normal GB velocity $\left(v_{n}\right)$ during shearcoupled motion. Our previous work further revealed that CTBs also follow this behavior (Eq. (6)) since they exhibit shear-coupled normal motion (Chen and Kulkarni, 2013). We note that although lipid membranes, high angle GBs, and CTBs display a different relationship between their fluctuation spectrum $A(k)$ and the wave vector $k$ (Eqs. (4), (5), and (6), respectively), they all essentially follow a power law with different exponents for $k$. 


\subsection{Fluctuation spectrum of a confined membrane}

The fact that a membrane fluctuating close to adjacent surfaces, such as in a multilayer system, experiences an effective repulsive pressure arising from confinement was first introduced by Helfrich (1978). The commonly used model can be represented by a simpler problem of modeling the fluctuations of a single membrane constrained by two rigid plates, as shown in Fig. 4. Contrary to an unconfined membrane, there is a constraint on the

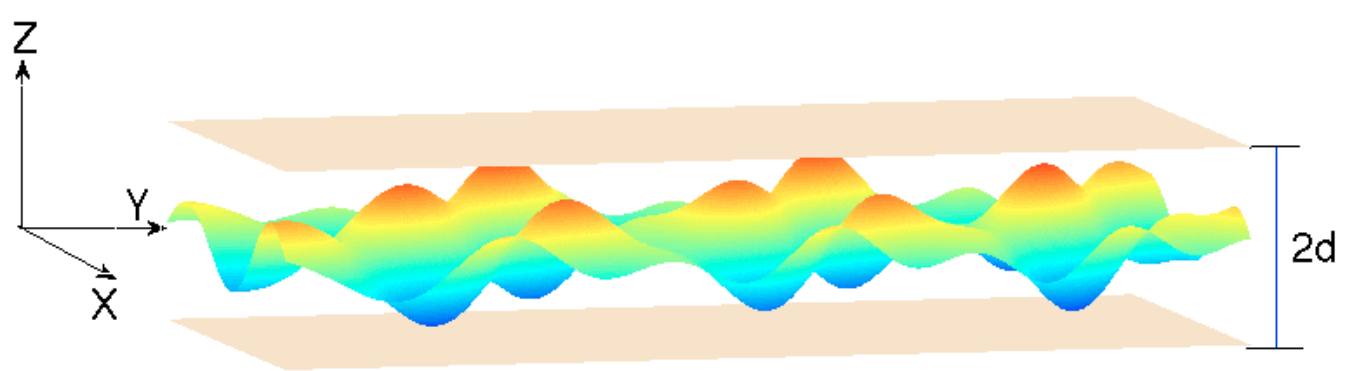

Figure 4: Schematic of a confined membrane fluctuating between two rigid plates.

out-of-plane deflection $h(x, y)$ that

$$
-d \leq h(x, y) \leq d
$$

where the rigid plates are separated by a distance $2 d$. Recognizing the difficulty of imposing this constraint inequality, many researchers (Radler et al., 1995; Gov et al., 2003; Merath and Siefert, 2007; Farago, 2008; Freund, 2012) proposed a 'soft constraint' to replace Eq. (8). Specifically, it is assumed that the net result of the confinement is that the membrane

experiences an effective harmonic potential that can be introduced as an additional term in the Hamiltonian. This modifies Eq. (1) to

$$
\mathcal{H}=b \int_{0}^{W} \frac{1}{2}\left[\kappa\left(h^{\prime \prime}(x)\right)^{2}+\gamma h^{2}(x)\right] d x
$$

where $\gamma$ is a constant that accounts for the confinement. Thus the mean square fluctuation spectrum becomes

$$
\left\langle A^{2}(k)\right\rangle=\frac{k_{B} T}{b W\left(\kappa k^{4}+\gamma\right)}
$$




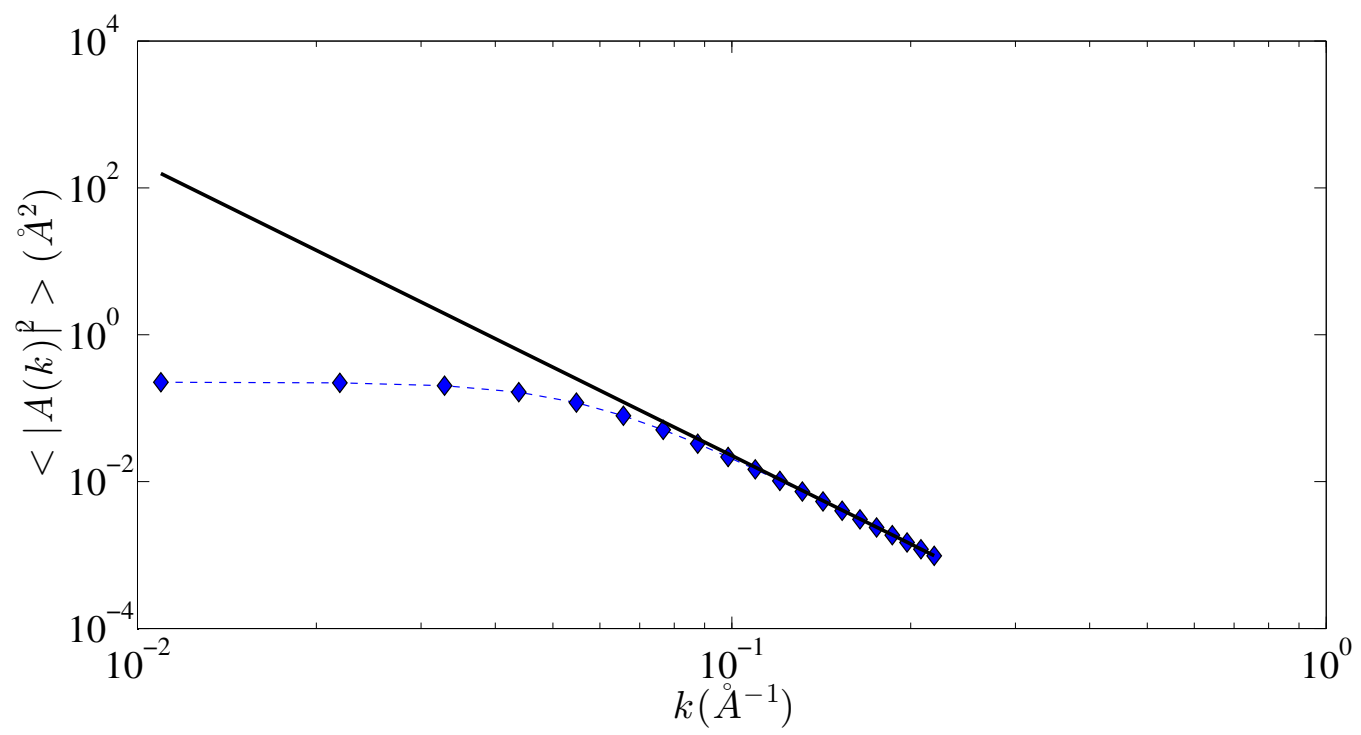

Figure 5: Plot of the fluctuation spectrum $\left\langle A^{2}(k)\right\rangle$ with respect to wave vector $k$ for a free (unconstrained) membrane (solid black line), and a membrane with confinement (dotted blue line). This plot is a representation of Eq. (10) and does not represent a real simulation.

It is evident that the effect of $\gamma$ can be ignored for small wavelength. However, at long wavelength or small $k, \gamma$ is the dominant term and the fluctuation spectrum becomes independent of $k$. This is represented in Fig. 5 which plots Eq. (10). This implies that the repulsive force between fluctuating membranes arising from the steric interactions inhibits their fluctuations especially in the long wavelength modes. This correlation between repulsive force and suppression of fluctuations also appears in the entropic interaction between solid-liquid interfaces (Hoyt et al., 2001) and crystalline membranes such as bilayer graphene (Zakharchenko et al., 2010). Thus, we conclude that membranes and interfaces whose free (unconstrained) fluctuations follow Eq. (4) or Eq. (5) exhibit a suppression of long wavelength fluctuations under confinement owing to the repulsive nature of entropic interactions. In the following sections, we examine the entropic interactions and effect on fluctuations of multiple CTBs, or in general, interfaces whose free fluctuations follow Eq. (6).

\section{Thermal fluctuations of multiple twin boundaries}

We first present our molecular dynamics simulations that examine the thermal fluctuations of multiple, parallel CTBs as a function of the twin boundary spacing. 


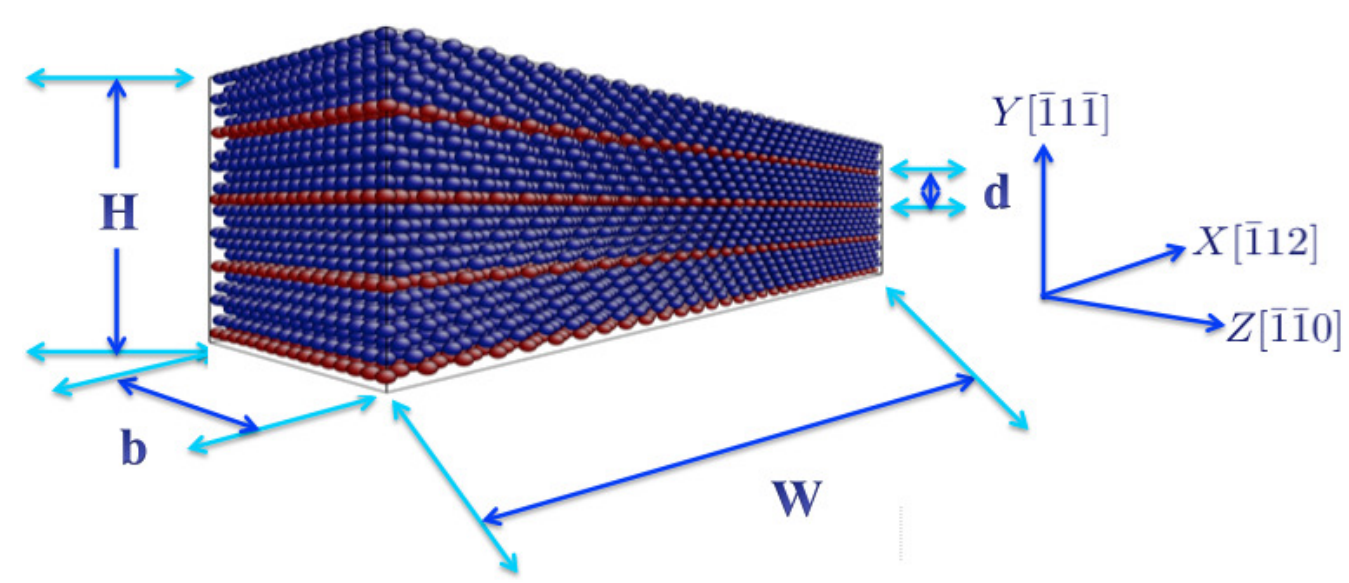

Figure 6: Atomistic structure of a nanotwinned specimen showing the crystallographic orientation. Equally spaced CTBs separated by distance $d$ are shown by red colored atoms. The rest of the face-centered-cubic atoms are shown in blue color.

\subsection{Simulation method}

The nanotwinned structures were modeled by way of molecular dynamics simulations using LAMMPS (Plimpton , 1995). All simulations were performed on Cu using the embeddedatom-method (EAM) interatomic potential developed by Mishin et al. (2001). As shown in Fig. 6, the specimen were oriented along the $[\overline{1} 12]$, $[\overline{1} 1 \overline{1}]$, and [ $\overline{1} \overline{1} 0]$ crystallographic directions. Thus, the Y-direction was aligned normal to the plane of the twin boundaries. The simulation cell dimensions in the lateral $(\mathrm{X}$ and $\mathrm{Z}$ ) directions were $W \approx 56 \mathrm{~nm}$, and $b \approx 1.9$ $\mathrm{nm}$, with periodic boundary conditions applied in all directions. Since the length in the Z-direction is much smaller than that in the $\mathrm{X}$-direction $(b \ll W)$, fluctuations along the Z-direction can be ignored. Thus the problem can be regarded as quasi-one dimensional. The CTB spacing was varied from $0.6 \mathrm{~nm}$ to $12 \mathrm{~nm}$. As shown in Fig. 7, each specimen with a different CTB spacing had a different height in the Y-direction since the number of CTBs was held fixed at $N=4$ (including one CTB located at the top/bottom end of the specimen due to periodicity).

The simulation cell was first relaxed at zero temperature using energy minimization to obtain an equilibrium configuration. Then the structure was equilibrated at $800 \mathrm{~K}$ for 10 ps under the NPT ensemble in order to allow for thermal expansion and to relax the thermal stresses in all directions. This was followed by equilibration for $0.5 \mathrm{~ns}$ under the NVT ensemble using the Nose-Hoover thermostat. The fluctuation spectrum for one of the 


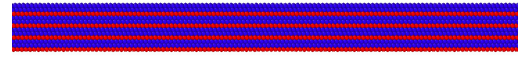

(a) $\mathrm{d}=0.6 \mathrm{~nm}$

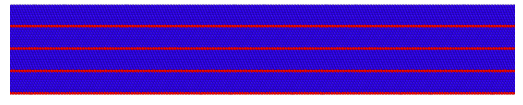

(b) $\mathrm{d}=1.8 \mathrm{~nm}$

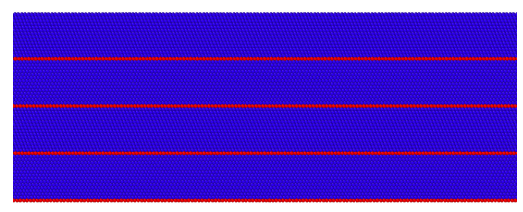

(c) $\mathrm{d}=3.0 \mathrm{~nm}$

Figure 7: Atomistic structure of a typical nanotwinned specimen with four CTB $(N=4)$ and different spacings $d$. The red atoms represent the CTBs.

CTBs was then extracted from the equilibrium simulations. The details of computing the fluctuation spectrum of a twin boundary using molecular dynamics simulations are described in our previous work (Chen and Kulkarni, 2013).

\subsection{Simulation results}

Fig. 8 shows the variation of the fluctuation spectrum of a CTB as a function of the wave vector $k$ in the presence of multiple parallel CTBs. For clarity, only the simulation results for $0.6 \mathrm{~nm}, 1.8 \mathrm{~nm}$, and $3.0 \mathrm{~nm}$ twin boundary spacings are shown. We first observe that in the presence of adjoining CTBs, the long wavelength fluctuation modes of CTBs are enhanced. This is in sharp contrast to other interfaces discussed earlier which exhibit a suppression of fluctuations. We further note that as the twin boundary spacing $d$ decreases, more fluctuation modes are affected (and enhanced). For example, for a twin boundary spacing of $0.6 \mathrm{~nm}$, the fluctuation with wavelength greater than $4 \mathrm{~nm}$ are amplified, whereas if the spacing is 3.0 $\mathrm{nm}$, only the wavelengths greater than $30 \mathrm{~nm}$ are amplified. Examining the mean square of the out-of-plane displacement in real space $\left\langle h^{2}\right\rangle$ also yields the same trend. Fig. 9 shows that as the twin boundary spacing decreases, the out-of-plane displacements of the CTBs are enhanced. The effect of neighboring CTBs is diminished beyond a CTB spacing of $3 \mathrm{~nm}$.

In order to verify whether this behavior is unique to CTBs and not a common characteristic of all solid-solid interfaces separated by a crystalline matrix, we also performed a series of molecular dynamics simulations on specimens containing multiple GBs, instead of CTBs, to extract the change in their fluctuations due to confinement. The simulation procedure was similar to that described above and the specimen consisted of parallel $\Sigma 5(310)$ GBs with periodic boundary conditions in all directions. We specifically selected a high-angle GB since they exhibit capillarity induced fluctuations under unconfined conditions as represented by 


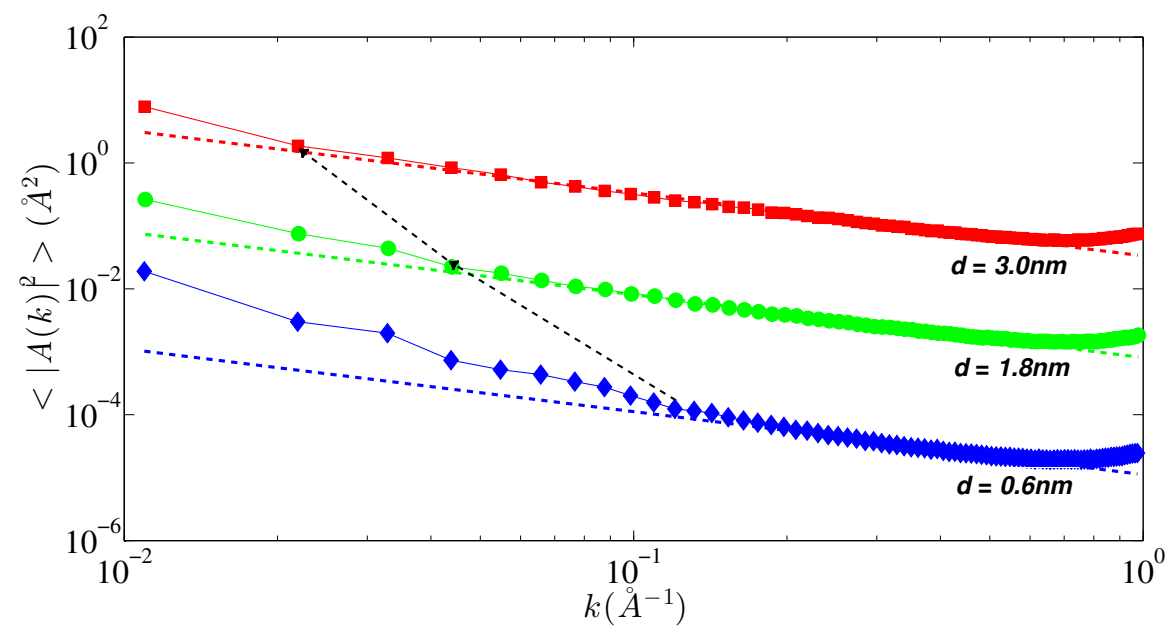

Figure 8: Fluctuation spectrum as a function of wave vector for different twin spacing $d$. The different curves have been shifted for a better comparison. Thus, the values of $\left\langle|A(k)|^{2}\right\rangle$ specified along the vertical axis do not represent the real values.

Eq. (5), unlike unconfined CTBs which follow Eq. (6). As expected, Fig. 10 shows that such high angle GBs experience a noticeable suppression of the long wavelength modes similar to fluid membranes. Thus, compared to the suppression of the fluctuation spectra of confined lipid membranes, solid-liquid interfaces and general high-angle GBs due to the repulsive nature of their entropic interactions, the obvious enhancement of the fluctuations in the case of CTBs indicates that there should exist an attractive entropic force between CTBs. In the following sections, we seek to verify this conjecture through statistical mechanics based analysis.

\section{Entropic interaction between fluctuating twin boundaries}

As mentioned above, since fluid membranes are flexible and undergo large fluctuations, they exert a repulsive force on each other as they come close. Starting with the pioneering work of Helfrich (1978), this repulsive steric interaction has been studied using the classical model of a membrane fluctuating between two rigid plates (Janke and Kleinert, 1987; Bachmann et al., 2001; Freund, 2012, 2013; Hanlumyuang et al., 2014). In contrast to fluid membranes, the twin boundaries are separated by a crystalline matrix which also undergoes deformation to accommodate the fluctuations of the neighboring interfaces. Specifically, the out-of-plane fluctuations of the CTBs are accommodated by localized shear-coupled normal 


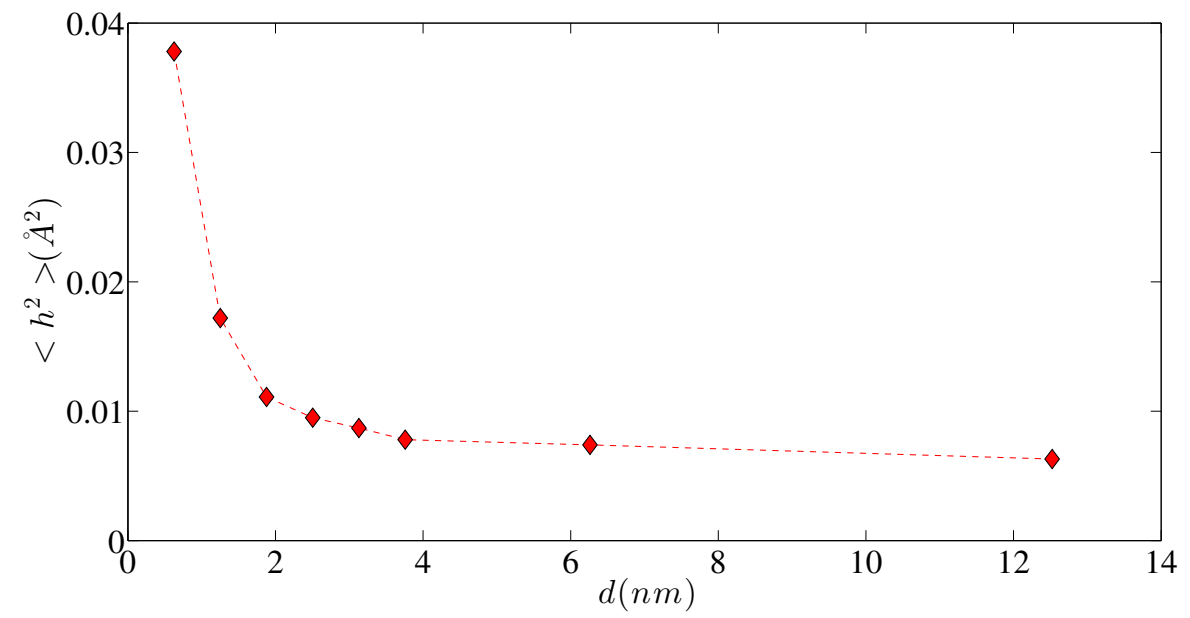

Figure 9: Mean square out-of-plane displacement $\left\langle h^{2}\right\rangle$ in real space as a function of twin spacing $d$.

motion which induces strain fields in the elastically deformable matrix (Chen and Kulkarni, 2013). Hence, the rigid plate model is not suitable for this case. Instead, we begin by formulating a linear elastic boundary value problem for two fluctuating CTBs and calculating the strain energy based on the continuum model proposed by Karma et al. (2012). We then calculate the associated Hemholtz free energy for this system as a function of the twin boundary spacing $2 d$, to finally obtain the entropic force. We note that the problem addressed here as well our approach is similar in some aspects to the statistical mechanics based study of interaction between fluctuating dislocations by Rickman and Lesar (2001). Interestingly, they also find that the entropic contribution to the force between dislocations at finite temperature is attractive.

\subsection{Boundary value problem}

Fig. 11(a) shows the schematic of two fluctuating twin boundaries (denoted by CTB-1 and CTB-2) under thermal equilibrium with their flat configurations located at distance $d$ above and below the $x$ axis respectively. Similar to our atomistic simulations, the length in the $x$ direction is denoted by $W$, and the thickness in the $z$ direction is denoted by $b$ and points out of the plane of the paper. With $b \ll W$, the problem is treated as quasi one-dimensional. We describe the out-of-plane displacements of the two CTBs by functions 


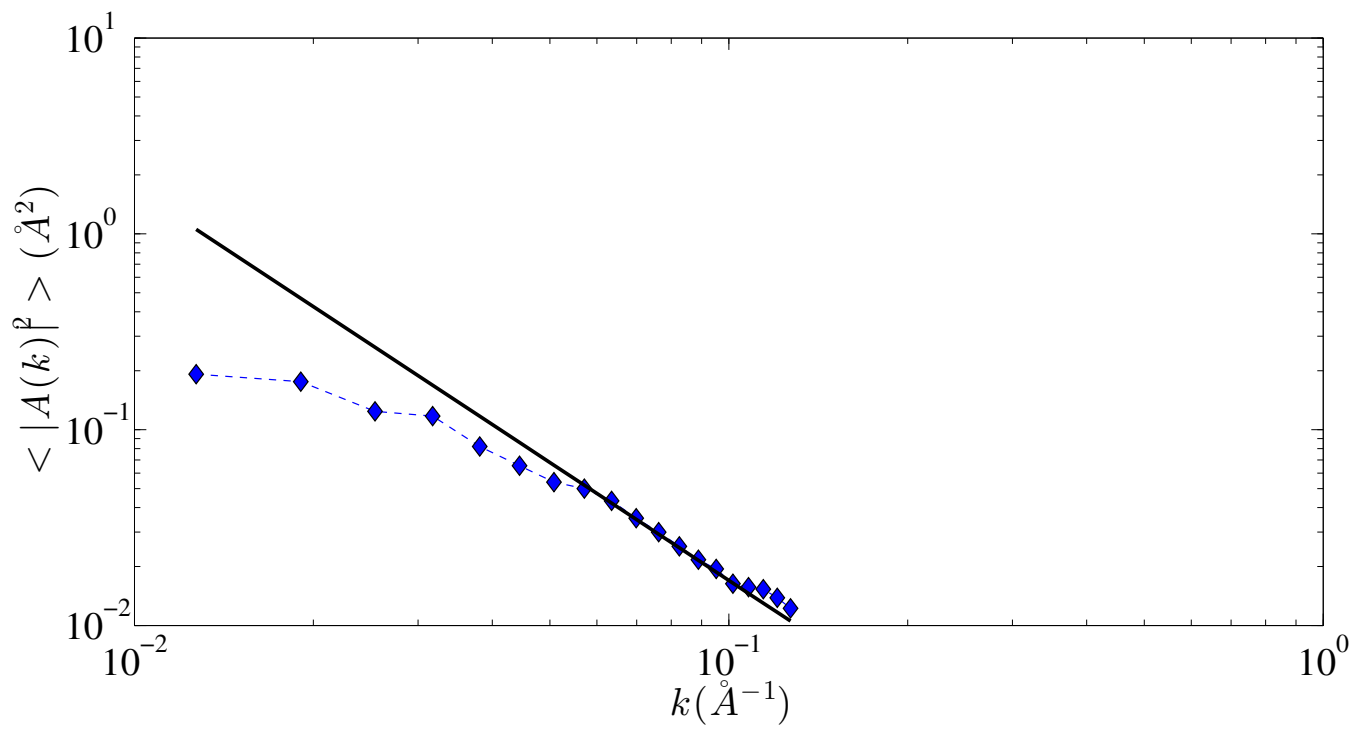

Figure 10: Fluctuation spectrum as a function of wave vector $k$ for a single $\Sigma 5(310)$ high-angle GB (solid line), and multiple GBs (dashed line). The solid line has a slope of -2 as expected from Eq. (5).

$h^{1}(x)$ and $h^{2}(x)$ which can be expanded in Fourier series as follows:

$$
\begin{aligned}
& h^{1}(x)=\sum_{i=0}^{N} \hat{h}_{i}^{1}=\sum_{i=0}^{N}\left[a_{i} \cos \left(k_{i} x\right)+c_{i} \sin \left(k_{i} x\right)\right], \\
& h^{2}(x)=\sum_{j=0}^{N} \hat{h}_{j}^{2}=\sum_{j=0}^{N}\left[b_{j} \cos \left(k_{j} x\right)+d_{j} \sin \left(k_{j} x\right)\right],
\end{aligned}
$$

where $N=\frac{W}{l_{0}}, l_{0}$ being the minimum lattice distance along the $x$ direction, $a_{i} \equiv a\left(k_{i}\right)$, $c_{i} \equiv c\left(k_{i}\right), b_{j} \equiv b\left(k_{j}\right), d_{j} \equiv d\left(k_{j}\right)$, and $k_{i}=\frac{2 \pi}{W} i$ is the wave vector. The first task is to obtain the strain energy, which we denote by $E\left(h^{1}, h^{2}\right)$, induced by these two perturbations $h^{1}(x)$ and $h^{2}(x)$. For simplicity, we first consider a pair of single cosine modes, as shown in Fig. 11(b). Due to the coupling relation, Eq. (7), the perturbations of both CTBs must induce shear strains in the adjoining grains, as shown in Fig. 11(c). Thus, the problem of computing the strain energy of a pair of CTBs fluctuating as cosine modes is reduced to calculating the associated strain field in the crystalline matrix. To this end, we first define three regions, namely, $U$ (upper) for $y \geq d, L$ (lower) for $y \leq-d$ and $M$ (middle) for $-d \leq y \leq d$ (Fig. 11(b)). Let $u$ and $v$ be the $x$ and $y$ components of the displacement vector respectively. The components of the displacement field in the three regions are denoted by $\left(u^{U}(x, y), v^{U}(x, y)\right)$, 


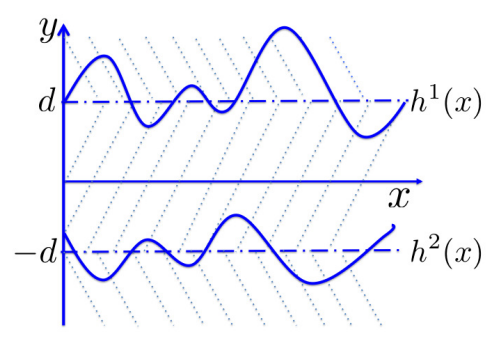

(a)

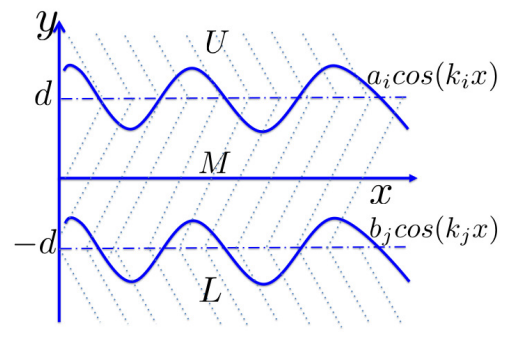

(b)

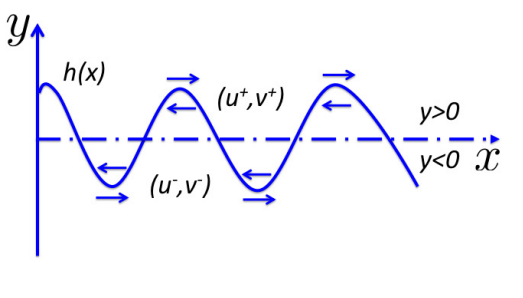

(c)

Figure 11: Schematic of the continuum model for interaction of CTBs. (a) Two CTBs (solid lines) fluctuating around their equilibrium positions with spacing $2 d$. The dashed lines indicate that the atomic orientations on either side of the CTBs are mirror images. (b) A pair of cosine mode fluctuations. We use $U$ (upper $), M($ middle $), L($ lower $)$ to denote the three regions separated by the CTBs. (c) A sinusoidal perturbation $h(x)$ of the CTB. Based on the model by Karma et al. (2012), the apparent normal (out-of-plane) displacement of the CTB is caused by relative tangential displacement (indicated by arrows) of atoms with respect to the neighboring atomic layers via lattice reorientation. $\left(u^{+}, v^{+}\right)$and $\left(u^{-}, v^{-}\right)$denote the $\mathrm{x}$ and $\mathrm{y}$ components of the displacement field above and below the unperturbed CTB.

$\left(u^{M}(x, y), v^{M}(x, y)\right)$ and $\left(u^{L}(x, y), v^{L}(x, y)\right)$. Assuming isotropic linear elasticity, these fields satisfy the following equilibrium equations:

$$
\begin{aligned}
& \left(\partial_{x}^{2}+\partial_{y}^{2}\right) u^{R}+\frac{1}{1-2 \nu} \partial_{x}\left(\partial_{x} u^{R}+\partial_{y} v^{R}\right)=0 \\
& \left(\partial_{x}^{2}+\partial_{y}^{2}\right) v^{R}+\frac{1}{1-2 \nu} \partial_{y}\left(\partial_{x} u^{R}+\partial_{y} v^{R}\right)=0
\end{aligned}
$$


with $\nu$ being the Poisson's ratio, and $R$ taking values $U, M$, and $L$, subject to the following boundary conditions:

$$
\begin{array}{r}
u^{U}(x, d)-u^{M}(x, d)=\beta h^{1}(x)=\beta a_{i} \cos \left(k_{i} x\right) \\
u^{M}(x,-d)-u^{L}(x,-d)=-\beta h^{2}(x)=-\beta b_{j} \cos \left(k_{j} x\right) \\
v^{U}(x, d)=v^{M}(x, d) \\
v^{M}(x,-d)=v^{L}(x,-d) \\
\sigma_{y y}^{U}(x, d)=\sigma_{y y}^{M}(x, d) \\
\sigma_{y y}^{M}(x,-d)=\sigma_{y y}^{L}(x,-d) \\
\sigma_{x y}^{U}(x, d)=\sigma_{x y}^{M}(x, d) \\
\sigma_{x y}^{M}(x,-d)=\sigma_{x y}^{L}(x,-d) \\
u^{U}(x, \infty)=u^{L}(x,-\infty)=v^{U}(x, \infty)=v^{L}(x,-\infty)=0
\end{array}
$$

Eq. (13a) and Eq. (13b) indicate that the normal displacements of the CTBs corresponding to $h^{1}(x)$ and $h^{2}(x)$ must be coupled to relative tangential translations of the adjoining regions of the matrix and twin by $\beta h^{1}(x)$ and $-\beta h^{2}(x)$ respectively. We should note here that the minus sign in Eq. (13b) signifies that these two CTBs have reversed crystallographic orientations of the grains above and below. Thus, the directions of the shear coupled normal motion experienced by CTB-1 and CTB-2 are reversed. Eq. (13c) and Eq. (13d) enforce the continuity of the normal displacement. Eqs. (13e), (13f), (13g), (13h) are continuity equations for the normal and shear components of the traction vector at the interfaces where the normal stress is given by

$$
\sigma_{y y}^{R}(x, y)=\lambda \partial_{x} u^{R}(x, y)+(\lambda+2 \mu) \partial_{y} v^{R}(x, y)
$$

and the shear stress is given by

$$
\sigma_{x y}^{R}(x, y)=\mu\left[\partial_{x} v^{R}(x, y)+\partial_{y} u^{R}(x, y)\right]
$$


Finally, Eqs. (13i) implement the far field boundary conditions. To circumvent the problem of directly solving the boundary value problem with these complicated boundary conditions, we decompose it into two simpler independent boundary value problems using the linearity of Eq. (12). Specifically, the complete displacement field is constructed by superimposing the displacement field induced by CTB-1 with perturbation $h^{1}(x)=a_{i} \cos \left(k_{i} x\right)$ and coupling coefficient $\beta$ located at $y=d$, and that induced by CTB-2 with perturbation $h^{2}(x)=$ $b_{j} \cos \left(k_{j} x\right)$ and coupling coefficient $-\beta$ located at $y=-d$ (Fig. 12). Karma et al. (2012)

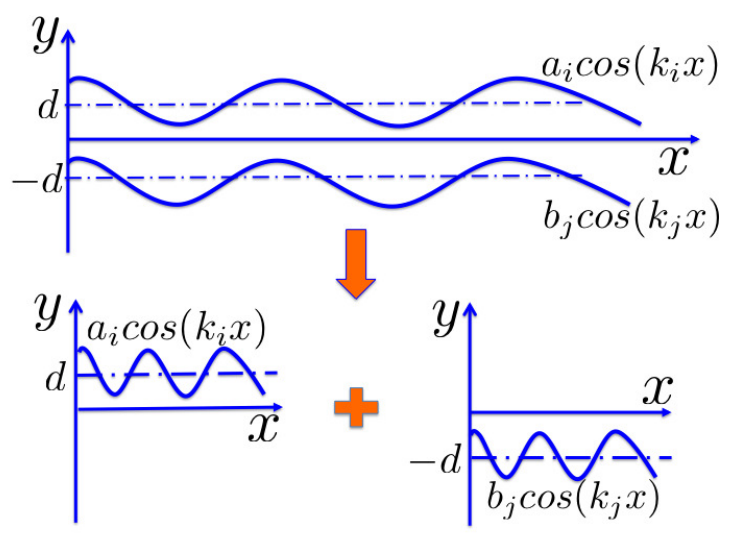

Figure 12: Decomposition of the complete displacement field due to two fluctuating CTBs into two simpler cases involving a single fluctuating CTB at different locations.

obtained the following displacement field induced by a perturbation $h(x)=a(k) \cos (k x)$ of an interface with coupling coefficient $\beta$ located by $y=0$, as shown in Fig. 11(c):

$$
\begin{array}{r}
u=\left[\frac{\beta a(k)}{2} \operatorname{sgn}(y)-\frac{\beta a(k)}{4(1-\nu)} k y\right] e^{-k|y|} \cos k x \\
v=\left[\frac{\beta a(k)}{4(1-\nu)}(1-2 \nu)+\frac{\beta a(k)}{4(1-\nu)} \operatorname{sgn}(y) k y\right] e^{-k|y|} \sin k x
\end{array}
$$

Translating this solution in order to obtain the displacement field induced by perturbations located at $y=d$ and $y=-d$ respectively, and then superimposing them, we have the complete displacement field as follows: 
In region $U$,

$$
\begin{array}{r}
u^{U}(x, y)=\frac{\beta a_{i}}{2}\left[1-\frac{k_{i}(y-d)}{2(1-\nu)}\right] e^{-k_{i}(y-d)} \cos k_{i} x+ \\
\frac{-\beta b_{j}}{2}\left[1-\frac{k_{j}(y+d)}{2(1-\nu)}\right] e^{-k_{j}(y+d)} \cos k_{j} x \\
v^{U}(x, y)=\frac{\beta a_{i}}{4(1-\nu)}\left[1-2 \nu+k_{i}(y-d)\right] e^{-k_{i}(y-d)} \sin k_{i} x+ \\
\frac{-\beta b_{j}}{4(1-\nu)}\left[1-2 \nu+k_{j}(y+d)\right] e^{-k_{j}(y+d)} \sin k_{j} x
\end{array}
$$

In region $M$,

$$
\begin{array}{r}
u^{M}(x, y)=\frac{-\beta a_{i}}{2}\left[1+\frac{k_{i}(y-d)}{2(1-\nu)}\right] e^{-k_{i}(y-d)} \cos k_{i} x+ \\
\frac{-\beta b_{j}}{2}\left[1-\frac{k_{j}(y+d)}{2(1-\nu)}\right] e^{-k_{j}(y+d)} \cos k_{j} x \\
v^{M}(x, y)=\frac{\beta a_{i}}{4(1-\nu)}\left[1-2 \nu-k_{i}(y-d)\right] e^{-k_{1}(y-d)} \sin k_{i} x+ \\
\frac{-\beta b_{j}}{4(1-\nu)}\left[1-2 \nu+k_{j}(y+d)\right] e^{-k_{j}(y+d)} \sin k_{j} x
\end{array}
$$

In region $L$,

$$
\begin{array}{r}
u^{L}(x, y)=\frac{-\beta a_{i}}{2}\left[1+\frac{k_{i}(y-d)}{2(1-\nu)}\right] e^{-k_{i}(y-d)} \cos k_{i} x+ \\
\frac{\beta b_{j}}{2}\left[1+\frac{k_{j}(y+d)}{2(1-\nu)}\right] e^{-k_{j}(y+d)} \cos k_{j} x \\
v^{L}(x, y)=\frac{\beta a_{i}}{4(1-\nu)}\left[1-2 \nu-k_{i}(y-d)\right] e^{-k_{i}(y-d)} \sin k_{i} x+ \\
\frac{-\beta b_{j}}{4(1-\nu)}\left[1-2 \nu-k_{j}(y+d)\right] e^{-k_{j}(y+d)} \sin k_{j} x
\end{array}
$$

\subsection{Strain Energy}

Having determined the displacement field, we can now calculate the total strain energy associated with the cosine mode perturbations of the two CTBs. Using the periodicity of 
the perturbations, $h^{1}(x)$, and $h^{2}(x)$, the strain energy is expressed as (Karma et al., 2012)

$E\left(a_{i}, b_{j}\right)=\frac{b W}{\Lambda}\left[\int_{0}^{\Lambda} d x \int_{d}^{\infty} e\left(u^{U}, v^{U}\right) d y+\int_{0}^{\Lambda} d x \int_{-d}^{d} e\left(u^{M}, v^{M}\right) d y+\int_{0}^{\Lambda} d x \int_{-\infty}^{-d} e\left(u^{L}, v^{L}\right) d y\right]$

where $\Lambda$ is introduced as the least common multiple of the wavelengths $2 \pi / k_{i}$ and $2 \pi / k_{j}$ of the fluctuations of CTB-1 and CTB-2, respectively. $W$ is assumed to be a large integer multiple of $\Lambda . e\left(u^{U}, v^{U}\right), e\left(u^{M}, v^{M}\right)$, and $e\left(u^{L}, v^{L}\right)$ are the elastic energy densities in the regions $U, M$, and $L$, respectively, and are given by

$$
e\left(u^{R}, v^{R}\right)=\mu \frac{1-\nu}{1-2 \nu}\left(\frac{\partial u^{R}}{\partial x}+\frac{\partial v^{R}}{\partial y}\right)^{2}+\frac{\mu}{2}\left(\frac{\partial u^{R}}{\partial y}+\frac{\partial v^{R}}{\partial x}\right)^{2}-2 \mu \frac{\partial u^{R}}{\partial x} \frac{\partial v^{R}}{\partial y}
$$

where, $R$ represents $U, M$, and $L$ respectively.

The integrals over $x$ are evaluated using the orthogonal relations

$$
\frac{1}{\Lambda} \int_{0}^{\Lambda} \sin \left(k_{i} x\right) \sin \left(k_{j} x\right) d x=\frac{1}{\Lambda} \int_{0}^{\Lambda} \cos \left(k_{i} x\right) \cos \left(k_{j} x\right) d x=\frac{1}{2} \delta_{i j}
$$

where $\delta_{i j}$ is the Kronecker delta, and

$$
\frac{1}{\Lambda} \int_{0}^{\Lambda} \sin \left(k_{i} x\right) \cos \left(k_{j} x\right) d x=\frac{1}{\Lambda} \int_{0}^{\Lambda} \cos \left(k_{i} x\right) \sin \left(k_{j} x\right) d x=0
$$

All the integrals over $y$ can also be computed analytically. Summing up the contributions after integration over both $x$ and $y$, we obtain

$$
E\left(a_{i}, b_{j}\right)=\frac{b W \beta^{2} \mu a_{i}^{2} k_{i}}{8(1-\nu)}+\frac{b W \beta^{2} \mu b_{j}^{2} k_{j}}{8(1-\nu)}-\delta_{i j} \frac{b W \beta^{2} \mu a_{i} b_{j} k_{i}^{2}}{4(1-\nu)} e^{-2 k_{i} d}\left(\frac{1}{k_{i}}-2 d\right)
$$

The first two terms consisting of $a_{i}^{2}$ and $b_{j}^{2}$ represent the contribution from the self-energy induced by independent fluctuations of the CTBs. The last term consisting of the product $a_{i} b_{j}$ represents the interaction energy between CTB-1 and CTB-2. Naturally, the separation distance $2 d$ enters the energy expression through the interaction term. We note that the Kronecker delta implies that there is non-zero interaction energy when the wave numbers for the two CTBs are the same. This is also consistent with the observation made by 
Rickman and Lesar (2001) in their study of fluctuating dislocation lines. Following the above calculations, the strain energy associated with CTB-1 and CTB-2 undergoing sine mode fluctuations, $c_{i} \sin \left(k_{i} x\right)$ and $d_{j} \sin \left(k_{j} x\right)$, respectively, can also be evaluated as

$$
E\left(c_{i}, d_{j}\right)=\frac{b W \beta^{2} \mu c_{i}^{2} k_{i}}{8(1-\nu)}+\frac{b W \beta^{2} \mu d_{j}^{2} k_{j}}{8(1-\nu)}-\delta_{i j} \frac{b W \beta^{2} \mu c_{i} d_{j} k_{i}^{2}}{4(1-\nu)} e^{-2 k_{i} d}\left(\frac{1}{k_{i}}-2 d\right)
$$

Finally, we consider the remaining two scenarios. When CTB-1 undergoes fluctuation $a_{i} \cos \left(k_{i} x\right)$, and CTB-2 undergoes fluctuation $d_{j} \sin \left(k_{j} x\right)$, or when CTB-1 undergoes fluctuation $c_{i} \sin \left(k_{i} x\right)$, and CTB-2 undergoes fluctuation $b_{j} \cos \left(k_{j} x\right)$, it can be seen that the interaction energy is zero owing to Eq. (23). We are now ready to evaluate the total strain energy, $E\left(h^{1}, h^{2}\right)$, as a sum over the contribution from the self-energy and interaction energy for all the modes. Thus, evaluating the Kronecker delta in Eqs. (24) and (25), we have

$$
\begin{aligned}
E\left(h^{1}, h^{2}\right) & =\sum_{i, j} E\left(\hat{h}_{i}^{1}, \hat{h}_{j}^{2}\right) \\
& =\sum_{i, j}\left[E\left(a_{i}, b_{j}\right)+E\left(c_{i}, d_{j}\right)\right] \\
& =\sum_{i}\left[\frac{b W \beta \mu a_{i}^{2} k_{i}}{8(1-\nu)}+\frac{b W \beta \mu b_{i}^{2} k_{i}}{8(1-\nu)}-\frac{b W \beta \mu a_{i} b_{i} k_{i}^{2}}{4(1-\nu)} e^{-2 k_{i} d}\left(\frac{1}{k_{i}}-2 d\right)\right. \\
& \left.+\frac{b W \beta \mu c_{i}^{2} k_{i}}{8(1-\nu)}+\frac{b W \beta \mu d_{i}^{2} k_{i}}{8(1-\nu)}-\frac{b W \beta \mu c_{i} d_{i} k_{i}^{2}}{4(1-\nu)} e^{-2 k_{i} d}\left(\frac{1}{k_{i}}-2 d\right)\right]
\end{aligned}
$$

\subsection{Helmholtz free energy and entropic force}

In order to obtain the Helmholtz free energy of the system, we first construct the partition function $Z_{i}$ for wave vector $k_{i}$, using the strain energy corresponding to the wave vector $k_{i}$

$$
\begin{aligned}
E_{i} & =\frac{b W \beta \mu a_{i}^{2} k_{i}}{8(1-\nu)}+\frac{b W \beta \mu b_{i}^{2} k_{i}}{8(1-\nu)}-\frac{b W \beta \mu a_{i} b_{i} k_{i}^{2}}{4(1-\nu)} e^{-2 k_{i} d}\left(\frac{1}{k_{i}}-2 d\right) \\
& +\frac{b W \beta \mu c_{i}^{2} k_{i}}{8(1-\nu)}+\frac{b W \beta \mu d_{i}^{2} k_{i}}{8(1-\nu)}-\frac{b W \beta \mu c_{i} d_{i} k_{i}^{2}}{4(1-\nu)} e^{-2 k_{i} d}\left(\frac{1}{k_{i}}-2 d\right)
\end{aligned}
$$

Assuming the coupling parameter $\beta \simeq 1$, and introducing the following normalization

$$
E_{0}=\frac{b W^{2} \mu}{8(1-\nu)}, A_{i}=\frac{a_{i}}{W}, B_{i}=\frac{b_{i}}{W}, C_{i}=\frac{c_{i}}{W}, D_{i}=\frac{d_{i}}{W}, K_{i}=k_{i} W, \varphi=\frac{2 d}{W},
$$


we get

$$
\begin{aligned}
E_{i} & =E_{0} K_{i} A_{i}^{2}+E_{0} K_{i} B_{i}^{2}-2 E_{0} K_{i} A_{i} B_{i} e^{-K_{i} \varphi}\left(1-K_{i} \varphi\right) \\
& +E_{0} K_{i} C_{i}^{2}+E_{0} K_{i} D_{i}^{2}-2 E_{0} K_{i} C_{i} D_{i} e^{-K_{i} \varphi}\left(1-K_{i} \varphi\right)
\end{aligned}
$$

Then, the partition function corresponding to the wave vector $k_{i}$ is obtained as

$$
\begin{aligned}
Z_{i} & =\int_{-\infty}^{\infty} d A_{i} \int_{-\infty}^{\infty} d B_{i} \int_{-\infty}^{\infty} d C_{i} \int_{-\infty}^{\infty} d D_{i} e^{-\frac{E_{i}}{k_{B} T}} \\
& =\left[\int_{-\infty}^{\infty} d A_{i} \int_{-\infty}^{\infty} d B_{i} e^{-\frac{E_{i A B}}{k_{B} T}}\right]^{2}
\end{aligned}
$$

where $E_{i A B}=E_{0} K_{i} A_{i}^{2}+E_{0} K_{i} B_{i}^{2}-2 E_{0} K_{i} A_{i} B_{i} e^{-K_{i} \varphi}\left(1-K_{i} \varphi\right)$. Let $P_{i}=\frac{E_{0} K_{i}}{k_{B} T}$ and $Q_{i}=$ $\frac{E_{0} K_{i}}{k_{B} T} e^{-K_{i} \varphi}\left(1-K_{i} \varphi\right)$. It is obvious that $P_{i}>Q_{i}>0$. Then

$$
\frac{E_{i A B}}{k_{B} T}=P_{i} A_{i}^{2}+P_{i} B_{i}^{2}-2 Q_{i} A_{i} B_{i}
$$

Introduce new variables $s_{i}=\sqrt{P} B_{i}+\sqrt{P} A_{i}, t_{i}=\sqrt{P} B_{i}-\sqrt{P} A_{i}$,

$$
\frac{E_{i A B}}{k_{B} T}=\frac{P_{i}-Q_{i}}{2 P_{i}} s_{i}^{2}+\frac{P_{i}+Q_{i}}{2 P_{i}} t_{i}^{2}
$$

Substituting into Eq. (27), we obtain

$$
\begin{aligned}
Z_{i} & =\left[\int_{-\infty}^{\infty} d s_{i} e^{-\frac{P_{i}-Q_{i}}{2 P_{i}} s_{i}^{2}} \int_{-\infty}^{\infty} d t_{i} e^{-\frac{P_{i}+Q_{i}}{2 P_{i}} t_{i}^{2}} \frac{1}{2 P_{i}}\right]^{2} \\
& =\frac{\pi^{2}}{P_{i}^{2}-Q_{i}^{2}}
\end{aligned}
$$

The complete partition function becomes

$$
Z=\prod_{i}^{N} Z_{i}=\pi^{2 N} \prod_{i}^{N} \frac{1}{P_{i}^{2}-Q_{i}^{2}}
$$


Then, the Helmholtz free energy is computed as

$$
F=-k_{B} T \ln Z=k_{B} T \sum_{i}^{N} \ln \left(P_{i}^{2}-Q_{i}^{2}\right)-2 N k_{B} T \ln \pi
$$

The entropic force can be defined as

$$
f=-\frac{\partial F}{\partial(2 d)}=-\frac{1}{W} \frac{\partial F}{\partial \varphi}
$$

Given the complexity of the summation, we evaluate Eq. (36) numerically. To this end, we use material properties for copper and other parameters that are consistent with our atomistic simulations: $W=56 \mathrm{~nm}, b=1.9 \mathrm{~m}, \mu=56.64 \mathrm{GPa}, T=800 \mathrm{~K}, \nu=0.25, l_{0}=4.4275 \AA$, $N=126 . l_{0}=\sqrt{6} a / 2($ where $a=3.615 \AA)$ is taken as the lattice parameter along the $x$ axis which corresponds to the [112] crystallographic direction. Fig. 13 shows the entropic force between fluctuating CTBs versus twin spacing $2 d$. It can be seen that the entropic interaction is indeed attractive and decays as $1 / d^{2}$.

$$
f \propto-\frac{1}{d^{2}}
$$

Comparing Fig. 9 and Fig. 13, it is somewhat surprising that an attractive force with a magnitude of just over $1 \mathrm{MPa}$ for the smallest CTB spacing of $0.6 \mathrm{~nm}$ can lead to almost a doubling of the fluctuation amplitude. Nevertheless, our theoretical analysis confirms our previous conjecture that the entropic force between CTBs must be attractive given the enhancement of the CTB fluctuations observed in atomistic simulations.

\section{Concluding Remarks}

In summary, we present a study of the interaction between twin boundaries at finite temperature using molecular dynamics simulations and statistical mechanics based on a continuum representation of a fluctuating twin boundary in an elastic medium. Our atomistic simulations reveal that as the spacing between twin boundaries decreases, their fluctuations are enhanced, and are almost doubled in the case of the smallest twin boundary spacing of $6 \AA$. This is in contrast to many other crystalline interfaces such as high angle grain 


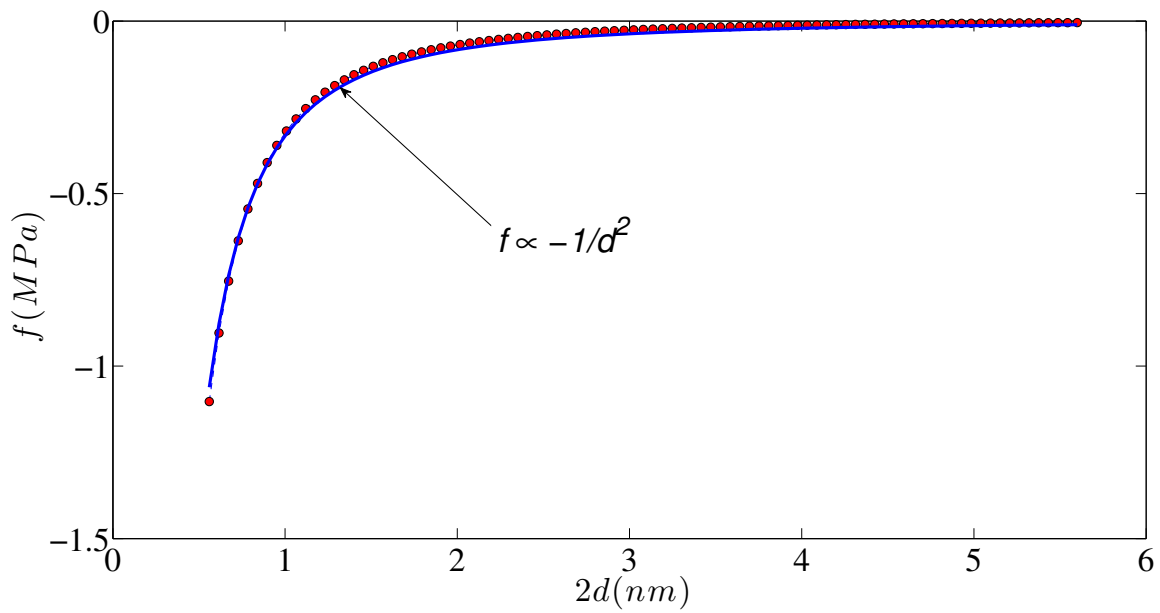

Figure 13: Entropic force as a function of twin spacing $2 d$. The blue line is the fitting curve which shows $1 / d^{2}$ dependence.

boundaries and crystal-melt interfaces, which exhibit a remarkable suppression of fluctuations in multilayer systems due to the repulsive nature of their entropic interactions. Our theoretical analysis of the entropic interaction of twin boundaries shows that there is indeed an attractive force between fluctuating twin boundaries which accounts for the increase in fluctuations observed in our simulations. The rather surprising attractive force is attributed to the characteristic of twin boundaries to exhibit shear-coupled normal motion. Since our continuum model for a fluctuating twin boundary follows the work of Karma et al. (2012) on the fluctuations of shear coupled grain boundaries, our findings should also be valid for low angle grain boundaries that exhibit shear-coupled normal motion. More generally, we conclude that for shear coupled grain boundaries, the entropic interaction is dominated by the deformation of the elastic medium between adjoining interfaces, and hence is fundamentally distinct from the entropic pressure resulting from steric hindrance. In fact, the entropic contribution to twin boundary interactions reported here is in qualitative agreement with the attractive nature of interactions between fluctuating dislocations at finite temperature observed by Rickman and Lesar (2001). A possible implication of this attractive force is that twin boundaries do not exhibit spontaneous migration away from each other, unlike high angle grain boundaries that are prone to migration. At the same time, owing to the small magnitude, the attractive force is not strong enough to pull adjacent twin boundaries closer to annihilate them. Taken together, our study suggests greater thermal stability 
of nanotwinned metals even with angstrom scale spacing of twin boundaries compared to nanocrystalline metals that are susceptible to grain growth, especially at high temperatures. These observations open avenues for further investigation through computations and possible experiments.

\section{Acknowledgement}

We gratefully acknowledge the support of NSF under grant CMMI-1129041. The sim-

ulations were performed on the supercomputing facility (Maxwell) hosted by the Research Computing Center at University of Houston. We thank Prof. Pradeep Sharma, Dr. Qian Deng and Dr. Yuranan Hanlumyuang for insightful discussions.

\section{References}

Bachmann, M., Kleinert, H., Peister, A., 2001. Fluctuation pressure of membrane between walls. Phy. Rev. E 63, 051709.

Bezares, J., Jiao, S., Liu, Y., Bufford, D., Lu, L., Zhang, X., Kulkarni, Y., Asaro, R.J., 2012. Indentation of Nano-twinned FCC metals: Implications for nano-twin stability. Acta Mater. 60, 4623.

Cahn, J.W., Mishin, Y., Suzuki, A., 2006. Coupling grain boundary motion to shear deformation. Acta Mater. 54, 4953.

Chen, D., Kulkarni, Y., 2013. Elucidating the kinetics of twin boundaries from thermal fluctuations. MRS. Commun. 4, 241.

Dao, M., Lu, L., Shen, Y.F., Suresh, S., 2006. Strength, strain-rate sensitivity and ductility of copper with nanoscale twins. Acta Mater. 54, 5421.

Dao, M., Lu, L., Asaro, R.J., De Hosson, J.T.M., Ma, E., 2007. Toward a quantitative understanding of mechanical behavior of nanocrystalline metals. Acta Mater. 55, 4041.

Demkowicz, M., Anderoglu, O., Zhang, X., Misra A., 2011. The influence of sigma 3 twin boundaries on the formation of radiation-induced defect clusters in nanotwinned $\mathrm{Cu}$. J. Maters. Res. 26, 1666. 
Farago, O., 2008. Membrane fluctuations near a plane rigid surface. Phys. Rev. E 78, 051919.

Freund, L.B., 2012. Fluctuation pressure on a bio-membrane confined within a parabolic potential well. Acta Mechanica Sinica 28, 1180.

Freund, L.B., 2013. Entropic pressure between biomembranes in a periodic stack due to thermal fluctuations. Proc. Natl. Acad. Sci. 110, 2047.

Gao, W., Huang, R., 2014. Thermomechanics of monolayer graphene: Rippling, thermal expansion and elasticity. J. Mech. Phys. Solids 66, 42.

Gleiter, H., 1989. Nanocrystalline Materials. Prog. Mater. Sci. 33, 223.

Gov, N., Zilman, A.G., Safran, S., 2003. Cytoskeleton confinement and tension of red blood cell membranes. Phys. Rev. Lett. 90, 228108.

Guo, X., Xia, Y., 2011. Repulsive force vs. source number: Competing mechanisms in the yield of twinned gold nanowires of finite length. Acta Mater. 59, 2350.

Hanlumyuang, Y., Liu, L.P., Sharma, P., 2014. Revisiting the entropic force between fluctuating biological membranes. J. Mech. Phys. Solids 63, 179.

Helfrich, W., 1973. Elastic properties of lipid bilayers: theory and possible experiments. Z. Naturforsch 28c, 693.

Helfrich, W., 1978. Steric interaction of fluid membranes in multilayer systems. Naturforsch A $33,241$.

Hodge, A.M., Wang, Y.M., Barbee Jr., T.W., 2008. Mechanical deformation of high-purity sputter-deposited nano-twinned copper. Scripta Mater. 59, 163.

Hoyt, J.J., Asta, M., Karma, A., 2001. Method for computing the anisotropy of the solidliquid interfacial free energy. Phys. Rev. Lett. 86, 5530.

Hoyt, J.J., Trautt, Z.T., Upmanyu, M., 2006. Interface mobility from interface random walk. Science 314, 632 . 
Hoyt, J.J., Trautt, Z.T., Upmanyu, M., 2010. Fluctuation in molecular dynamics simulation. Math. Comp. Simul. 80, 1382.

Jang, D., Li, X., Gao, H., Greer, J.R., 2012. Deformation mechanisms in nanotwinned metal nanopillars. Nature Nanotech. 7, 594.

Janke, W., Kleinert, H., 1987. Fluctuation pressure of a stack of membrane. Phys. Rev. Lett. 58, 144.

Karma, A., Trautt, Z.T., Mishin, Y., 2012. Relationship between Equilibrium Fluctuations and Shear-Coupled Motion of Grain Boundaries. Phys. Rev. Lett. 109, 095501.

Kulkarni, Y., Asaro, R.J., 2009. Are some nano-twinned FCC metals optimal for strength and grain stability. Acta Mater. 57, 4835.

Kulkarni, Y., Asaro, R.J., Farkas, D., 2009. Are nanotwinned structures in fcc metals optimal for strength, ductility and grain stability? Scripta Mater. 60, 532.

Li, X., Wei, Y., Lu, L., Lu, K., Gao, H., 2010. Dislocation nucleation governed softening and maximum strength in nanotwinned metals. Nature 464, 877.

Los, J.H., Katsnelson, M.I., Yazyev, O.V., Zakharchenko, K.V., Fasolino, A., 2009. Scaling properties of flexible membranes from atomistic simulations: application to graphene. Phys. Rev. B 80, 121405.

Lu, L., Shen, Y., Chen, X., Qian, L., Lu, K., 2004. Ultrahigh strength and high electrical conductivity in copper. Science 304, 422.

Lu, K., Lu, L., Suresh, S., 2009. Strengthening materials by engineering coherent internal boundaries at the nanoscale. Science 324, 349.

Merath, R.J., Siefert, U., 2007. Fluctuation spectra of free and supported membrane pairs. European Phys. Journal E 23, 103.

Mirkhani, H., Joshi, S.P., 2014. Mechanism-based crystal plasticity modeling of twin boundary migration in nanotwinned face-centered-cubic metals. J. Mech. Phys. Solids 68, 107. 
Mishin, Y., Mehl, M.J., Papaconstantopoulos, D.A., Voter, A.F., Kress, J.D., 2001. Structural stability and lattice defects in copper: Ab initio, tight-binding, and embedded-atom calculations. Phys. Rev. B 63, 224106.

Nelson, D.R., Piran, T., Weinberg, S., 2004. Statistical Mechanics of Membranes and Surfaces. World Scientific Publishing Co., Singapore.

Plimpton, S.J., 1995. Fast parallel algorithms for short-range molecular dynamics. J. Comp. Phys. 117, 1.

Radler, J.O., Feder, T.J., Strey, H.H., Sackmann, E., 1995. Mechanics of ultra-strength materials. Phys. Rev. E 51, 4526.

Rickman, J.M., Lesar, R., 2001. Finite-temperature dislocation interactions. Phy. Rev. B 64, 094106.

Safran, S.A., 2003. Statistical Thermodynamics Of Surfaces, Interfaces, And Membranes. Westview Press, Colorado.

Wang, J., Sansoz, F., Huang, J., Liu, Y., Sun, S., Zhang, Z., Mao, S.X., 2013. Near-ideal theoretical strength in gold nanowires containing angstrom scale twins. Nature Commun. 4,1742 .

Wright, T.W., Daphalapurkar, N.P., Ramesh, K.T., 2014. Stability of ideal fcc twin boundaries. J. Mech. Phys. Solids 73, 228.

Yu, K.Y., Bufford, D., Sun, C., Liu, Y., Wang, H., Kirk, M.A., Li, M., Zhang, X., 2013. Removal of stacking-fault tetrahedra by twin boundaries in nanotwinned metals. Nature Commun., 4, 1377.

Zakharchenko, K.V., Los, J.H., Katsnelson, M.I., Fasolino, A., 2010 Atomistic simulations of structural and thermodynamic properties of bilayer graphene. Phys. Rev. B 81, 235439.

Zhang, X., Wang, H., Chen, X.H., Lu, L., Lu, K., Hoagland, R.G., Misra, A., 2006. Highstrength sputter-deposited $\mathrm{Cu}$ foils with preferred orientation of nanoscale growth twins. Appl. Phys. Lett. 88, 173116. 
Zhu, T., Li, J., Ogata, S., Yip, S., 2009. Mechanics of ultra-strength materials. MRS Bulletin 34, 167. 